\title{
Pointwise approximation by a Durrmeyer variant of Bernstein-Stancu operators
}

Lvxiu Dong and Dansheng Yu*

"Correspondence:

dsyu@hznu.edu.cn

Department of Mathematics,

Hangzhou Normal University,

Hangzhou, Zhejiang 310036, China

\section{Abstract}

In the present paper, we introduce a kind of Durrmeyer variant of Bernstein-Stancu operators, and we obtain the direct and converse results of approximation by the operators.

MSC: 41A25; 41A35

Keywords: Bernstein-Stancu type operators; pointwise and global estimates; inverse results

\section{Introduction}

For any $f \in C_{[0,1]}$, the corresponding Bernstein operators and Bernsetin-Durrmeyer operators are defined by

$$
B_{n}(f, x):=\sum_{k=0}^{n} f\left(\frac{k}{n}\right) p_{n k}(x)
$$

and

$$
D_{n}(f, x):=(n+1) \sum_{k=0}^{n} p_{n k}(x) \int_{0}^{1} f(t) p_{n k}(t) d t,
$$

respectively, where $p_{n k}(x):=\left(\begin{array}{l}n \\ k\end{array}\right) x^{k}(1-x)^{n-k}, k=0,1, \ldots, n$. Both $B_{n}(f, x)$ and $D_{n}(f, x)$ have played very important roles in approximation theory and computer science. There are many generalizations of the operators $B_{n}(f, x)$ and $D_{n}(f, x)$. Among them, Gadjiev and Ghorbanalizadeh [1] introduced the following new generalized Bernstein-Stancu type operators with shifted knots:

$$
S_{n, \alpha, \beta}(f, x):=\left(\frac{n+\beta_{2}}{n}\right)^{n} \sum_{k=0}^{n} f\left(\frac{k+\alpha_{1}}{n+\beta_{1}}\right) q_{n k}(x)
$$

where $x \in A_{n}:=\left[\frac{\alpha_{2}}{n+\beta_{2}}, \frac{n+\alpha_{2}}{n+\beta_{2}}\right]$, and

$$
q_{n k}(x):=\left(\begin{array}{l}
n \\
k
\end{array}\right)\left(x-\frac{\alpha_{2}}{n+\beta_{2}}\right)^{k}\left(\frac{n+\alpha_{2}}{n+\beta_{2}}-x\right)^{n-k}, \quad k=0,1, \ldots, n,
$$

(c) The Author(s) 2017. This article is distributed under the terms of the Creative Commons Attribution 4.0 International License (http://creativecommons.org/licenses/by/4.0/), which permits unrestricted use, distribution, and reproduction in any medium, provided you give appropriate credit to the original author(s) and the source, provide a link to the Creative Commons license, and indicate if changes were made. 
with $\alpha_{k}, \beta_{k}, k=1,2$ positive numbers satisfying $0 \leq \alpha_{1} \leq \beta_{1}, 0 \leq \alpha_{2} \leq \beta_{2}$. Obviously, when $\alpha_{1}=\alpha_{2}=\beta_{1}=\beta_{2}=0, S_{n, \alpha, \beta}(f, x)$ reduces to the classical Bernstein operators in (1.1), when $\alpha_{2}=\beta_{2}=0$, it reduces to the so-called Bernstein-Stancu operators which were introduced by Stancu [2]:

$$
B_{n, \alpha, \beta}(f, x):=\sum_{k=0}^{n} f\left(\frac{k+\alpha}{n+\beta}\right) p_{n k}(x) .
$$

Some approximation properties and generalizations of the operators $S_{n, \alpha, \beta}(f, x)$ can be found in [3-5].

Motivated by (1.3), we introduce the following generalization of the operators (1.2):

$$
\widetilde{S}_{n, \alpha, \beta}(f, x):=\left(\frac{n+\beta_{2}}{n}\right)^{n} \sum_{k=0}^{n} \lambda_{n k}^{-1} q_{n k}(x) \int_{A_{n}} q_{n k}(t) f\left(\frac{n t+\alpha_{1}}{n+\beta_{1}}\right) d t,
$$

where

$$
\lambda_{n k}=\int_{A_{n}} q_{n k}(t) d t, \quad k=0,1, \ldots, n,
$$

and $\alpha_{k}, \beta_{k}, k=1,2$ positive numbers satisfying $0 \leq \alpha_{1} \leq \beta_{1}, 0 \leq \alpha_{2} \leq \beta_{2}$.

By Lemma 1 in Section 2 , we observe that $\widetilde{S}_{n, \alpha, \beta}(f, x)$ can be rewritten as follows:

$$
\widetilde{S}_{n, \alpha, \beta}(f, x)=\left(\frac{n+\beta_{2}}{n}\right)^{2 n+1} \sum_{k=0}^{n} q_{n k}(x)(n+1) \int_{A_{n}} q_{n k}(t) f\left(\frac{n t+\alpha_{1}}{n+\beta_{1}}\right) d t .
$$

Especially, when $\alpha_{1}=\alpha_{2}=\beta_{1}=\beta_{2}=0, \widetilde{S}_{n, \alpha, \beta}(f, x)$ reduces to the classical BernsteinDurrmeyer operators in (1.2). Many authors have studied some special cases of the operators $\widetilde{S}_{n, \alpha, \beta}(f, x)$. For example, the case $\alpha_{1}=\alpha_{2}=\beta_{1}=0$ in [6] by Jung, Deo, and Dhamija, the case $\alpha_{1}=\beta_{1}=0$ in [7] by Acar, Aral, and Gupta.

The main purpose of the present paper is to establish pointwise direct and converse approximation theorems of approximation by $\widetilde{S}_{n, \alpha, \beta}(f, x)$. To state our result, we need some notations:

$$
\begin{aligned}
& \omega_{\varphi^{\lambda}}^{2}(f, t)=\sup _{0<h \leq t} \sup _{x \pm h \varphi^{\lambda} \in A_{n}}\left|\Delta_{h \varphi^{\lambda}}^{2} f(x)\right|, \\
& D_{\lambda}^{2}=\left\{f \in C\left(A_{n}\right), f^{\prime} \in A \cdot C \cdot \mathrm{loc},\left\|\varphi^{2 \lambda} f^{\prime \prime}\right\|<+\infty\right\}, \\
& K_{\varphi^{\lambda}}\left(f, t^{2}\right)=\inf _{g \in D_{\lambda}^{2}}\left\{\|f-g\|+t^{2}\left\|\varphi^{2 \lambda} g^{\prime \prime}\right\|\right\}, \\
& \bar{D}_{\lambda}^{2}=\left\{f \in D_{\lambda}^{2},\left\|f^{\prime \prime}\right\|<+\infty\right\}, \\
& \bar{K}_{\varphi^{\lambda}}\left(f, t^{2}\right)=\inf _{g \in \bar{D}_{\lambda}^{2}}\left\{\|f-g\|+t^{2}\left\|\varphi^{2 \lambda} g^{\prime \prime}\right\|+t^{4 /(2-\lambda)}\left\|g^{\prime \prime}\right\|\right\},
\end{aligned}
$$

and $\varphi(x)=\sqrt{\left(x-\frac{\alpha_{2}}{n+\beta_{2}}\right)\left(\frac{n+\alpha_{2}}{n+\beta_{2}}-x\right)}, 0 \leq \lambda \leq 1$. It is well known (see [8], Theorem 3.1.2) that

$$
\omega_{\varphi^{\lambda}}^{2}(f, t) \sim K_{\varphi^{\lambda}}\left(f, t^{2}\right) \sim \bar{K}_{\varphi^{\lambda}}\left(f, t^{2}\right),
$$

where $x \sim y$ means that there exists a positive constant $c$ such that $c^{-1} y \leq x \leq c y$. 
Our first result can be read as follows.

Theorem 1 Let $f$ be a continuous function on $A_{n}, \lambda \in[0,1]$ be a fixed positive number. Then there exists a positive constant $C$ only depending on $\lambda, \alpha_{1}, \alpha_{2}, \beta_{1}$, and $\beta_{2}$ such that

$$
\left|\widetilde{S}_{n, \alpha, \beta}(f, x)-f(x)\right| \leq C\left(\omega_{\varphi^{\lambda}}\left(f, \frac{\delta_{n}^{1-\lambda}(x)}{\sqrt{n}}\right)+\omega\left(f, \frac{1}{n}\right)\right),
$$

where $\delta_{n}(x)=\varphi(x)+1 / \sqrt{n} \sim \max \{\varphi(x), 1 / \sqrt{n}\}$, and $\omega(f, t)$ is the usual modulus of continuity off on $A_{n}$.

Throughout the paper, $C$ denotes either a positive absolute constant or a positive constant that may depend on some parameters but not on $f, x$, and $n$. Their values may be different at different locations.

For the converse result, we have the following.

Theorem 2 Let $f$ be a continuous function on $A_{n}, 0<\alpha<\frac{2}{2-\lambda}, 0 \leq \lambda \leq 1$. Then

$$
\left|\widetilde{S}_{n, \alpha, \beta}(f, x)-f(x)\right|=O\left(\left(n^{-1 / 2} \delta_{n}^{1-\lambda}(x)\right)^{\alpha}\right)
$$

implies that

$$
\text { (i) } \quad \omega_{\varphi^{\lambda}}^{2}(f, t)=O\left(t^{\alpha}\right) ; \quad \text { (ii) } \quad \omega(f, t)=O\left(t^{\alpha(1-\lambda / 2)}\right) \text {. }
$$

\section{Auxiliary lemmas}

\section{Lemma 1 We have}

$$
\lambda_{k n}=\int_{A_{n}} q_{n k}(t) d t=\left(\frac{n}{n+\beta_{2}}\right)^{n+1} \frac{1}{n+1}, \quad k=0,1, \ldots, n .
$$

Proof For $p, q=1,2, \ldots$, set

$$
\begin{aligned}
B^{*}(p, q) & :=\int_{A_{n}}\left(x-\frac{\alpha_{2}}{n+\beta_{2}}\right)^{p-1}\left(\frac{n+\alpha_{2}}{n+\beta_{2}}-x\right)^{q-1} d x \\
& =\int_{0}^{\frac{n}{n+\beta_{2}}} x^{p-1}\left(\frac{n}{n+\beta_{2}}-x\right)^{q-1} d x
\end{aligned}
$$

Then

$$
\begin{aligned}
B^{*}(p, q) & =\frac{q-1}{p} \int_{0}^{\frac{n}{n+\beta_{2}}} x^{p}\left(\frac{n}{n+\beta_{2}}-x\right)^{q-2} d x \\
& =\frac{q-1}{p} \int_{0}^{\frac{n}{n+\beta_{2}}}\left(\frac{n}{n+\beta_{2}} x^{p-1}-x^{p-1}\left(\frac{n}{n+\beta_{2}}-x\right)\right)\left(\frac{n}{n+\beta_{2}}-x\right)^{q-2} d x \\
& =\frac{q-1}{p} \cdot \frac{n}{n+\beta_{2}} B^{*}(p, q-1)-\frac{q-1}{p} B^{*}(p, q),
\end{aligned}
$$

which implies that

$$
B^{*}(p, q)=\frac{q-1}{p+q-1} \cdot \frac{n}{n+\beta_{2}} B^{*}(p, q-1) .
$$


Therefore,

$$
\begin{aligned}
\lambda_{k n} & =\left(\begin{array}{l}
n \\
k
\end{array}\right) B^{*}(k+1, n-k+1) \\
& =\left(\frac{n}{n+\beta_{2}}\right)^{n-k}\left(\begin{array}{l}
n \\
k
\end{array}\right) \frac{(n-k)(n-k-1) \cdots 2 \cdot 1}{(n+1) n \cdots(k+2)} B^{*}(k+1,1) \\
& =\left(\frac{n}{n+\beta_{2}}\right)^{n-k} \frac{k+1}{(n+1)} \int_{0}^{\frac{n}{n+\beta_{2}}} x^{k} d x \\
& =\left(\frac{n}{n+\beta_{2}}\right)^{n+1} \frac{1}{n+1} .
\end{aligned}
$$

Lemma 2 For any $x \in A_{n}$, we have

$$
\widetilde{S}_{n, \alpha, \beta}\left((t-x)^{2}, x\right) \leq \frac{C}{n} \delta_{n}^{2}(x)
$$

Proof Write

$$
\widetilde{D}_{n, \alpha, \beta}(f, x):=\left(\frac{n+\beta_{2}}{n}\right)^{2 n+1} \sum_{k=0}^{n} q_{n k}(x)(n+1) \int_{A_{n}} q_{n k}(t) f(t) d t .
$$

Then [7]

$$
\begin{aligned}
\widetilde{D}_{n, \alpha, \beta}(1, x)=1, & \widetilde{D}_{n, \alpha, \beta}(t, x)=\frac{n}{n+2} x+\frac{n+2 \alpha_{2}}{(n+2)\left(n+\beta_{2}\right)}, \\
\widetilde{D}_{n, \alpha, \beta}\left(t^{2}, x\right)= & \left(x-\frac{\alpha_{2}}{n+\beta_{2}}\right)^{2} \frac{n(n-1)}{(n+2)(n+3)} \\
& +\frac{n}{n+\beta_{2}}\left(x-\frac{\alpha_{2}}{n+\beta_{2}}\right) \frac{4 n}{(n+2)(n+3)} \\
& +\left(\frac{n}{n+\beta_{2}}\right)^{2} \frac{2}{(n+2)(n+3)}+\frac{2 n \alpha_{2}}{(n+2)\left(n+\beta_{2}\right)}\left(x-\frac{\alpha_{2}}{n+\beta_{2}}\right) \\
& +\frac{2 n \alpha_{2}}{(n+2)\left(n+\beta_{2}\right)^{2}}+\left(\frac{\alpha_{2}}{n+\beta_{2}}\right)^{2},
\end{aligned}
$$

and

$$
\widetilde{D}_{n, \alpha, \beta}\left((t-x)^{2}, x\right) \leq \frac{C}{n} \delta_{n}^{2}(x) .
$$

By the facts that

$$
\begin{aligned}
& \widetilde{S}_{n, \alpha, \beta}(1, x)=\widetilde{D}_{n, \alpha, \beta}(1, x)=1, \\
& \widetilde{S}_{n, \alpha, \beta}(t, x)=\frac{n}{n+\beta_{1}} \widetilde{D}_{n, \alpha, \beta}(t, x)+\frac{\alpha_{1}}{n+\beta_{1}},
\end{aligned}
$$

and

$$
\widetilde{S}_{n, \alpha, \beta}\left(t^{2}, x\right)=\frac{n^{2}}{\left(n+\beta_{1}\right)^{2}} \widetilde{D}_{n, \alpha, \beta}\left(t^{2}, x\right)+\frac{2 n \alpha_{1}}{\left(n+\beta_{1}\right)^{2}} \widetilde{D}_{n, \alpha, \beta}(t, x)+\frac{\alpha_{1}^{2}}{\left(n+\beta_{1}\right)^{2}},
$$


we get

$$
\begin{aligned}
\widetilde{S}_{n, \alpha, \beta}\left((t-x)^{2}, x\right)= & \frac{n^{2}}{\left(n+\beta_{1}\right)^{2}} \widetilde{D}_{n, \alpha, \beta}\left((t-x)^{2}, x\right) \\
& +\left(\frac{2 n^{2} x}{\left(n+\beta_{1}\right)^{2}}+\frac{2 n \alpha_{1}}{\left(n+\beta_{1}\right)^{2}}-\frac{2 n x}{n+\beta_{1}}\right) \widetilde{D}_{n, \alpha, \beta}(t, x) \\
& +\frac{\alpha_{1}^{2}}{\left(n+\beta_{1}\right)^{2}}-\frac{2 \alpha_{1} x}{n+\beta_{1}}+x^{2}-\frac{n^{2}}{\left(n+\beta_{1}\right)^{2}} x^{2} \\
= & \frac{n^{2}}{\left(n+\beta_{1}\right)^{2}} \widetilde{D}_{n, \alpha, \beta}\left((t-x)^{2}, x\right)+\frac{\left(\beta_{1}^{2}+4 \beta_{1}\right) n+2 \beta_{1}^{2}}{\left(n+\beta_{1}\right)^{2}(n+2)} x^{2} \\
& +\frac{2 \alpha_{1}\left(\beta_{1}+\beta_{2}+2\right) n^{2}+2 n \alpha_{1}\left(\beta_{1} \beta_{2}+2 \beta_{1}+2 \beta_{2}\right)+4 \alpha_{1} \beta_{1} \beta_{2}}{\left(n+\beta_{1}\right)^{2}(n+2)\left(n+\beta_{2}\right)} x \\
& +\frac{\alpha_{1}^{2}}{\left(n+\beta_{1}\right)^{2}} \\
\leq & \widetilde{D}_{n, \alpha, \beta}\left((t-x)^{2}, x\right)+\frac{C}{n^{2}} \\
\leq & \frac{C}{n} \delta_{n}^{2}(x) .
\end{aligned}
$$

Lemma 3 For any given $\gamma \geq 0$, we have

$$
\sum_{k=0}^{n}\left|\frac{k+\alpha_{2}}{n+\beta_{2}}-x\right|^{\gamma}\left|q_{n k}(x)\right| \leq C \frac{\delta_{n}^{\gamma}(x)}{n^{\gamma / 2}}, \quad x \in[0,1] .
$$

Proof It was showed in [3] that

$$
\sum_{k=0}^{n}\left|\frac{k+\alpha_{1}}{n+\beta_{1}}-x\right|^{\gamma}\left|q_{n k}(x)\right| \leq C \frac{\left(\delta_{n}^{*}(x)\right)^{\gamma}}{n^{\gamma / 2}}, \quad x \in[0,1],
$$

where $\delta_{n}^{*}(x):=\psi(x)+\frac{1}{\sqrt{n}}$ and $\psi(x)=\sqrt{x(1-x)}$. We verify that

$$
\delta_{n}^{*}(x) \sim \delta_{n}(x), \quad x \in[0,1]
$$

In fact, when $x \in\left[\frac{2 \alpha_{2}+1}{n+\beta_{2}}, \frac{n-\beta_{2}+2 \alpha_{2}}{n+\beta_{2}}\right]$, we have

$$
\begin{aligned}
& \frac{1}{2} x \leq x-\frac{\alpha_{2}}{n+\beta_{2}} \leq x \\
& \frac{1}{2}(1-x) \leq \frac{n+\alpha_{2}}{n+\beta_{2}}-x \leq 1-x .
\end{aligned}
$$

Thus,

$$
\psi(x) \sim \varphi(x)
$$

which implies (2.7) for $x \in\left[\frac{2 \alpha_{2}+1}{n+\beta_{2}}, \frac{n-\beta_{2}+2 \alpha_{2}}{n+\beta_{2}}\right]$. When $x \in\left[0, \frac{2 \alpha_{2}+1}{n+\beta_{2}}\right) \cup\left(\frac{n-\beta_{2}+2 \alpha_{2}}{n+\beta_{2}}, 1\right]$, we have

$$
\delta_{n}^{*}(x) \sim \delta_{n}(x) \sim \frac{1}{\sqrt{n}}
$$

and thus (2.7) also holds. 
Now, by (2.6) and (2.7), we have

$$
\begin{aligned}
\sum_{k=0}^{n}\left|\frac{k+\alpha_{2}}{n+\beta_{2}}-x\right|^{\gamma}\left|q_{n k}(x)\right| & \leq \sum_{k=0}^{n}\left|\frac{k+\alpha_{2}}{n+\beta_{2}}-\frac{k+\alpha_{1}}{n+\beta_{1}}\right|^{\gamma}\left|q_{n k}(x)\right|+\sum_{k=0}^{n}\left|\frac{k+\alpha_{1}}{n+\beta_{1}}-x\right|^{\gamma}\left|q_{n k}(x)\right| \\
& \leq \frac{C}{n^{\gamma}} \sum_{k=0}^{n}\left|q_{n k}(x)\right|+C \frac{\delta_{n}^{\gamma}(x)}{n^{\gamma / 2}} \\
& \leq C \frac{\delta_{n}^{\gamma}(x)}{n^{\gamma / 2}}
\end{aligned}
$$

Lemma 4 For any $x \in A_{n}$, we have

$$
\sum_{k=0}^{n} q_{n k}(x)(n+1) \int_{A_{n}} \delta_{n}^{2}(t) q_{n k}(t) d t \leq C \delta_{n}^{2}(x)
$$

and

$$
\sum_{k=0}^{n-1} q_{n-1, k}(x) n \int_{A_{n}} \delta_{n}^{-2}(t) q_{n+1, k+1}(t) d t \leq C \delta_{n}^{-2}(x) .
$$

Proof By a similar calculation to that of Lemma 1, we have

$$
\int_{A_{n}} \varphi^{2}(t) q_{n k}(t) d t=\left(\frac{n}{n+\beta_{2}}\right)^{n+3} \frac{(n-k+1)(k+1)}{(n+3)(n+2)(n+1)} .
$$

On the other hand, we have

$$
\begin{aligned}
\sum_{k=0}^{n}\left(\frac{k}{n}-\frac{k^{2}}{n^{2}}\right) q_{n k}(x)= & \left(\frac{n}{n+\beta_{2}}\right)^{n-1}\left(x-\frac{\alpha_{2}}{n+\beta_{2}}\right)-\left(\frac{n}{n+\beta_{2}}\right)^{n-1} \frac{\left(x-\frac{\alpha_{2}}{n+\beta_{2}}\right)}{n} \\
& -\frac{n-1}{n}\left(\frac{n}{n+\beta_{2}}\right)^{n-2}\left(x-\frac{\alpha_{2}}{n+\beta_{2}}\right)^{2} \\
= & \frac{n-1}{n}\left(\frac{n}{n+\beta_{2}}\right)^{n-2} \varphi^{2}(x) .
\end{aligned}
$$

Therefore,

$$
\begin{aligned}
\sum_{k=0}^{n} q_{n k}(x)(n+1) \int_{A_{n}} \delta_{n}^{2}(t) q_{n k}(t) d t \leq & 2 \sum_{k=0}^{n} q_{n k}(x)(n+1) \int_{A_{n}}\left(\varphi^{2}(t)+\frac{1}{n}\right) q_{n k}(t) d t \\
\leq & 2 \sum_{k=0}^{n} q_{n k}(x)\left(\frac{n}{n+\beta_{2}}\right)^{n+3} \frac{(n-k+1)(k+1)}{(n+3)(n+2)} \\
& +\frac{C}{n} \sum_{k=0}^{n} q_{n k}(x) \\
\leq & C \sum_{k=0}^{n} q_{n k}(x)\left(\frac{(n-k) k}{n^{2}}+\frac{1}{n}\right)+\frac{C}{n} \\
\leq & C \delta_{n}^{2}(x),
\end{aligned}
$$

which proves (2.9). 
By Lemma 1, we have

$$
\begin{aligned}
n \int_{A_{n}} \delta_{n}^{-2}(t) q_{n+1, k+1}(t) d t & \leq \operatorname{Cn} \int_{A_{n}}\left(\varphi^{-2}(t)+n\right) q_{n+1, k+1}(t) d t \\
& \leq C n\left(\int_{A_{n}} \varphi^{-2}(t) q_{n+1, k+1}(t) d t+1\right) \\
& =\operatorname{Cn}\left(\frac{(n+1) n}{(k+1)(n-k)} \int_{A_{n}} q_{n-1, k}(t) d t+1\right) \\
& \leq C n\left(\frac{(n+1)}{(k+1)(n-k)}+1\right) \\
& \leq C n .
\end{aligned}
$$

Then

$$
\begin{aligned}
\sum_{k=0}^{n-1} q_{n-1, k}(x) n \int_{A_{n}} \delta_{n}^{-2}(t) q_{n+1, k+1}(t) d t & \leq C n \sum_{k=0}^{n} q_{n-1, k}(x) \\
& =C n \leq C \delta_{n}^{-2}(x) .
\end{aligned}
$$

Hence, (2.10) is proved.

Lemma 5 Iff is $r$ times differentiable on $[0,1]$, then

$$
\begin{aligned}
\widetilde{S}_{n, \alpha, \beta}^{(r)}(f, x)= & \left(\frac{n+\beta_{2}}{n}\right)^{2 n+1}\left(\frac{n}{n+\beta_{1}}\right)^{r} \frac{(n+1) ! n !}{(n-r) !(n+r) !} \sum_{k=0}^{n-r} q_{n-r, k}(x) \\
& \times \int_{\frac{\alpha_{2}}{n+\beta_{2}}}^{\frac{n+\alpha_{2}}{n+\beta_{2}}} q_{n+r, k+r}(t) f^{(r)}\left(\frac{n t+\alpha_{1}}{n+\beta_{1}}\right) d t .
\end{aligned}
$$

Proof By using Leibniz's theorem, we have

$$
\begin{aligned}
\widetilde{S}_{n, \alpha, \beta}^{(r)}(f, x)= & \left(\frac{n+\beta_{2}}{n}\right)^{2 n+1} \sum_{i=0}^{r} \sum_{k=i}^{n-r+i}\left(\begin{array}{l}
r \\
i
\end{array}\right) \frac{(-1)^{r-i}(n+1) !}{(k-i) !(n-k-r+i) !} \\
& \times\left(x-\frac{\alpha_{2}}{n+\beta_{2}}\right)^{k-i}\left(\frac{n+\alpha_{2}}{n+\beta_{2}}-x\right)^{n-k-r+i} \int_{\frac{\alpha_{2}}{n+\beta_{2}}}^{\frac{n+\alpha_{2}}{n+\beta_{2}}} q_{n k}(t) f\left(\frac{n t+\alpha_{1}}{n+\beta_{1}}\right) d t \\
= & \left(\frac{n+\beta_{2}}{n}\right)^{2 n+1} \sum_{k=i}^{n-r+i} \sum_{i=0}^{r}\left(\begin{array}{l}
r \\
i
\end{array}\right) \frac{(-1)^{r-i}(n+1) !}{(n-r) !} q_{n-r, k-i}(x) \\
& \times \int_{\frac{\alpha_{2}}{n+\beta_{2}}}^{\frac{n+\alpha_{2}}{n+\beta_{2}}} q_{n k}(t) f\left(\frac{n t+\alpha_{1}}{n+\beta_{1}}\right) d t \\
= & \left(\frac{n+\beta_{2}}{n}\right)^{2 n+1} \frac{(n+1) !}{(n-r) !} \sum_{k=0}^{n-r}(-1)^{r} q_{n-r, k}(x) \\
& \times \int_{\frac{\alpha_{2}}{n+\beta_{2}}}^{\frac{n+\alpha_{2}}{n+\beta_{2}}} \sum_{i=0}^{r}\left(\begin{array}{l}
r \\
i
\end{array}\right)(-1)^{i} q_{n, k+i}(t) f\left(\frac{n t+\alpha_{1}}{n+\beta_{1}}\right) d t .
\end{aligned}
$$


Since

$$
\frac{d^{r}}{d t^{r}} q_{n+r, k+r}(t)=\sum_{i=0}^{r}\left(\begin{array}{l}
r \\
i
\end{array}\right)(-1)^{i} \frac{(n+r) !}{n !} q_{n, k+i}(t),
$$

we have

$$
\begin{aligned}
\widetilde{S}_{n, \alpha, \beta}^{(r)}(f, x)= & \left(\frac{n+\beta_{2}}{n}\right)^{2 n+1} \frac{(n+1) ! n !}{(n-r) !(n+r) !} \sum_{k=0}^{n-r} q_{n-r, k}(x) \\
& \times \int_{\frac{\alpha_{2}}{n+\beta_{2}}}^{\frac{n+\alpha_{2}}{n+\beta_{2}}}(-1)^{r} q_{n+r, k+r}^{(r)}(t) f\left(\frac{n t+\alpha_{1}}{n+\beta_{1}}\right) d t .
\end{aligned}
$$

We obtain the required result by integrating by parts $r$ times.

Set

$$
\begin{aligned}
& \|f\|_{0}=\sup _{x \in A_{n}}\left\{\left|\delta_{n}^{\alpha(\lambda-1)}(x) f(x)\right|\right\} ; \\
& C_{\alpha, \lambda}=\left\{f \in C\left(A_{n}\right),\|f\|_{0}<+\infty\right\} ; \\
& \|f\|_{1}=\sup _{x \in A_{n}}\left\{\left|\delta_{n}^{\left(\frac{2}{2-\lambda}-\alpha\right)(1-\lambda)}(x) f^{\prime}(x)\right|\right\} ; \\
& C_{\alpha, \lambda}^{1}=\left\{f \in C_{\alpha, \lambda},\|f\|_{1}<+\infty\right\} ; \\
& \|f\|_{2}=\sup _{x \in A_{n}}\left\{\left|\delta_{n}^{2+\alpha(\lambda-1)}(x) f^{\prime \prime}(x)\right|\right\} ; \\
& C_{\alpha, \lambda}^{2}=\left\{f \in C_{\alpha, \lambda}, f^{\prime} \in A \cdot C \cdot \mathrm{loc}_{\mathrm{oc}},\|f\|_{2}<+\infty\right\} ; \\
& K_{\alpha, \lambda}^{1}(f, t)=\inf _{g \in C_{\alpha, \lambda}^{1}}\left\{\|f-g\|_{0}+t\|g\|_{1}\right\} ; \\
& K_{\alpha, \lambda}^{2}(f, t)=\inf _{g \in C_{\alpha, \lambda}^{2}}\left\{\|f-g\|_{0}+t\|g\|_{2}\right\} .
\end{aligned}
$$

Lemma 6 If $0 \leq \lambda \leq 1,0<\alpha<2$, then

$$
\begin{aligned}
& \left\|\widetilde{S}_{n, \alpha, \beta}(f)\right\|_{1} \leq C n^{1 /(2-\lambda)}\|f\|_{0}, \quad f \in C_{\alpha, \lambda}, \\
& \left\|\widetilde{S}_{n, \alpha, \beta}(f)\right\|_{1} \leq C\|f\|_{1}, \quad f \in C_{\alpha, \lambda}^{1} .
\end{aligned}
$$

Proof Firstly, we prove (2.13) by considering the following two cases.

Case 1. $x \in B_{n}:=\left[\frac{\alpha_{2}+1}{n+\beta_{2}}, \frac{n+\alpha_{2}-1}{n+\beta_{2}}\right]$. In this case, we have

$$
\varphi(x) \geq \min \left(\varphi\left(\frac{\alpha_{2}+1}{n+\beta_{2}}\right), \varphi\left(\frac{n+\alpha_{2}-1}{n+\beta_{2}}\right)\right) \geq \frac{C}{\sqrt{n}}
$$

which means that

$$
\delta_{n}(x) \sim \varphi(x) \quad \text { for } x \in B_{n} .
$$


By simple calculations, we have

$$
q_{n k}^{\prime}(x)=n \varphi^{-2}(x)\left(\frac{k+\alpha_{2}}{n+\beta_{2}}-x\right) q_{n k}(x)
$$

and

$$
\begin{aligned}
\delta_{n}\left(\frac{n t+\alpha_{1}}{n+\beta_{1}}\right) & =\sqrt{\left(t-\frac{\alpha_{2}}{n+\beta_{2}}+\frac{\alpha_{1}-\beta_{1} t}{n+\beta_{1}}\right)\left(\frac{n+\alpha_{2}}{n+\beta_{2}}-t+\frac{\beta_{1} t-\alpha_{1}}{n+\beta_{1}}\right)}+\frac{1}{\sqrt{n}} \\
& =\sqrt{\varphi^{2}(t)+O\left(\frac{1}{n}\right)}+\frac{1}{\sqrt{n}} \sim \varphi(t)+\frac{1}{\sqrt{n}}=\delta_{n}(t) .
\end{aligned}
$$

By (2.1), (2.15)-(2.17), and Hölder's inequality, we have

$$
\begin{aligned}
\left|\delta_{n}^{\left(\frac{2}{2-\lambda}-\alpha\right)(1-\lambda)}(x) \widetilde{S}_{n, \alpha, \beta}^{\prime}(f, x)\right| & \\
\leq & C n \varphi^{\left(\frac{2}{2-\lambda}-\alpha\right)(1-\lambda)-2}(x)\left(\frac{n+\beta_{2}}{n}\right)^{2 n+1} \\
\quad & \times \sum_{k=0}^{n} q_{n k}(x)\left|\frac{k+\alpha_{2}}{n+\beta_{2}}-x\right|(n+1)\left|\int_{A_{n}} f\left(\frac{n t+\alpha_{1}}{n+\beta_{1}}\right) q_{n k}(t) d t\right| \\
\leq & C n\|f\|_{0} \varphi^{\left(\frac{2}{2-\lambda}-\alpha\right)(1-\lambda)-2}(x) \sum_{k=0}^{n} q_{n k}(x)\left|\frac{k+\alpha_{2}}{n+\beta_{2}}-x\right|(n+1)\left|\int_{A_{n}} \delta_{n}^{\alpha(1-\lambda)}(t) q_{n k}(t) d t\right| \\
\leq & C n\|f\|_{0} \varphi^{\left(\frac{2}{2-\lambda}-\alpha\right)(1-\lambda)-2}(x) \sum_{k=0}^{n} q_{n k}(x)\left|\frac{k+\alpha_{2}}{n+\beta_{2}}-x\right|\left((n+1) \int_{A_{n}} \delta_{n}^{2}(t) q_{n k}(t) d t\right)^{\alpha(1-\lambda) / 2} \\
& \times\left((n+1) \int_{A_{n}} q_{n k}(t) d t\right)^{1-\alpha(1-\lambda) / 2} \\
\leq & C n\|f\|_{0} \varphi^{\left(\frac{2}{2-\lambda}-\alpha\right)(1-\lambda)-2}(x) \sum_{k=0}^{n} q_{n k}(x)\left|\frac{k+\alpha_{2}}{n+\beta_{2}}-x\right|\left((n+1) \int_{A_{n}} \delta_{n}^{2}(t) q_{n k}(t) d t\right)^{\alpha(1-\lambda) / 2} .
\end{aligned}
$$

By (2.9), (2.15) (2.5), and Hölder's inequality again, we have

$$
\begin{aligned}
& \left|\delta_{n}^{\left.\frac{2}{2-\lambda}-\alpha\right)(1-\lambda)}(x) \widetilde{S}_{n, \alpha, \beta}^{\prime}(f, x)\right| \\
& \leq C n\|f\|_{0} \varphi^{\left(\frac{2}{2-\lambda}-\alpha\right)(1-\lambda)-2}(x)\left(\sum_{k=0}^{n} q_{n k}(x)\left|\frac{k+\alpha_{2}}{n+\beta_{2}}-x\right|^{\frac{1}{1-\alpha(1-\lambda) / 2}}\right)^{1-\alpha(1-\lambda) / 2} \\
& \quad \times\left(\sum_{k=0}^{n} q_{n k}(x)(n+1) \int_{A_{n}} \delta_{n}^{2}(t) q_{n k}(t) d t\right)^{\alpha(1-\lambda) / 2} \\
& \leq C n^{1 / 2}\|f\|_{0} \varphi^{\frac{2(1-\lambda)}{2-\lambda}-1}(x) \leq C n^{1 /(2-\lambda)}\|f\|_{0} .
\end{aligned}
$$

Case 2. $x \in B_{n}^{c}=\left[\frac{\alpha_{2}}{n+\beta_{2}}, \frac{\alpha_{2}+1}{n+\beta_{2}}\right) \cup\left(\frac{n+\alpha_{2}-1}{n+\beta_{2}}, \frac{n+\alpha_{2}}{n+\beta_{2}}\right]$. In this case, we have

$$
\delta_{n}(x) \sim \frac{1}{\sqrt{n}}, \quad x \in B_{n}^{c}
$$


Noting that

$$
q_{n k}^{\prime}(x)=n\left(q_{n-1, k-1}(x)-q_{n-1, k}(x)\right)
$$

with $q_{n-1,-1}(x)=q_{n-1, n}(x)=0$, we get

$$
\widetilde{S}_{n, \alpha, \beta}^{\prime}(f, x)=n \sum_{k=0}^{n-1} q_{n-1, k}(x)(x)(n+1) \int_{A_{n}} f\left(\frac{n t+\alpha_{1}}{n+\beta_{1}}\right)\left(q_{n, k+1}(t)-q_{n, k}(t)\right) d t .
$$

Then, by using (2.17) and Hölder's inequality twice,

$$
\begin{aligned}
& \left|\delta_{n}^{\left(\frac{2}{2-\lambda}-\alpha\right)(1-\lambda)}(x) \widetilde{S}_{n, \alpha, \beta}^{\prime}(f, x)\right| \\
& \quad \leq C n \delta_{n}^{\left(\frac{2}{2-\lambda}-\alpha\right)(1-\lambda)}(x)\|f\|_{0}\left|\sum_{k=0}^{n-1} q_{n-1, k}(x)(n+1) \int_{A_{n}} \delta_{n}^{\alpha(1-\lambda)}(t)\left(q_{n, k+1}(t)+q_{n, k}(t)\right) d t\right| \\
& \quad \leq C n \delta_{n}^{\left(\frac{2}{2-\lambda}-\alpha\right)(1-\lambda)}(x)\|f\|_{0} \sum_{k=0}^{n-1} q_{n-1, k}(x)\left((n+1) \int_{A_{n}} \delta_{n}^{2}(t)\left(q_{n, k+1}(t)+q_{n, k}(t)\right) d t\right)^{\frac{\alpha(1-\lambda)}{2}} \\
& \quad \leq C n \delta_{n}^{\left(\frac{2}{2-\lambda}-\alpha\right)(1-\lambda)}(x)\|f\|_{0}\left(\sum_{k=0}^{n-1} q_{n-1, k}(x)(n+1) \int_{A_{n}} \delta_{n}^{2}(t)\left(q_{n, k+1}(t)+q_{n, k}(t)\right) d t\right)^{\frac{\alpha(1-\lambda)}{2}} \\
& \quad \leq C n \delta_{n}^{\left(\frac{2}{2-\lambda}-\alpha\right)(1-\lambda)}(x)\|f\|_{0} \delta_{n}^{\alpha(1-\lambda)} \\
& \quad \leq C n \frac{1}{2-\lambda}\|f\|_{0},
\end{aligned}
$$

where in the fourth inequality, we used the following fact, which can be deduced exactly in the same way as (2.10):

$$
\sum_{k=0}^{n-1} q_{n-1, k}(x)(n+1) \int_{A_{n}} \delta_{n}^{2}(t) q_{n k^{*}}(t) d t \leq C \delta_{n}^{2}(x) .
$$

We obtain (2.13) by combining (2.18) and (2.20).

Now, we begin to prove (2.14). If $\left(\frac{2}{2-\lambda}-\alpha\right)(\lambda-1)<0$, by (2.12) and using Hölder's inequality twice, we get

$$
\begin{aligned}
& \left|\delta_{n}^{\left(\frac{2}{2-\lambda}-\alpha\right)(1-\lambda)}(x) \widetilde{S}_{n, \alpha, \beta}^{\prime}(f, x)\right| \\
& \leq C\|f\|_{1}\left|\delta_{n}^{\left(\frac{2}{2-\lambda}-\alpha\right)(1-\lambda)}(x) n \sum_{k=0}^{n-1} q_{n-1, k}(x) \int_{A_{n}} q_{n+1, k+1}(t) \delta_{n}^{\left(\frac{2}{2-\lambda}-\alpha\right)(\lambda-1)}(t) d t\right| \\
& \leq C\|f\|_{1} \delta_{n}^{\left(\frac{2}{2-\lambda}-\alpha\right)(1-\lambda)}(x) \sum_{k=0}^{n-1} q_{n-1, k}(x)\left(n \int_{A_{n}} q_{n+1, k+1}(t) \delta_{n}^{-2}(t) d t\right)^{\frac{1}{2}\left(\frac{2}{2-\lambda}-\alpha\right)(1-\lambda)} \\
& \quad \times\left(n \int_{A_{n}} q_{n+1, k+1}(t) d t\right)^{1-\frac{1}{2}\left(\frac{2}{2-\lambda}-\alpha\right)(1-\lambda)} \\
& \leq C\|f\|_{1} \delta_{n}^{\left(\frac{2}{2-\lambda}-\alpha\right)(1-\lambda)}(x) \sum_{k=0}^{n-1} q_{n-1, k}(x)\left(n \int_{A_{n}} q_{n+1, k+1}(t) \delta_{n}^{-2}(t) d t\right)^{\frac{1}{2}\left(\frac{2}{2-\lambda}-\alpha\right)(1-\lambda)}
\end{aligned}
$$

(by $(2.1))$ 


$$
\begin{aligned}
& \leq C\|f\|_{1} \delta_{n}^{\left(\frac{2}{2-\lambda}-\alpha\right)(1-\lambda)}(x)\left(\sum_{k=0}^{n-1} q_{n-1, k}(x) n \int_{A_{n}} q_{n+1, k+1}(t) \delta_{n}^{-2}(t) d t\right)^{\frac{1}{2}\left(\frac{2}{2-\lambda}-\alpha\right)(1-\lambda)} \\
& \leq C\|f\|_{1}
\end{aligned}
$$

where, in the last inequality, (2.10) is applied.

If $\left(\frac{2}{2-\lambda}-\alpha\right)(\lambda-1)>0$, by using (2.9) instead of (2.10), we also can deduce that

$$
\left|\delta_{n}^{\left(\frac{2}{2-\lambda}-\alpha\right)(1-\lambda)}(x) \widetilde{S}_{n, \alpha, \beta}^{\prime}(f, x)\right| \leq C\|f\|_{1} .
$$

Lemma 7 If $0 \leq \lambda \leq 1,0<\alpha<2$, then

$$
\begin{aligned}
& \left\|\widetilde{S}_{n, \alpha, \beta}(f)\right\|_{2} \leq C n\|f\|_{0}, \quad f \in C_{\alpha, \lambda}, \\
& \left\|\widetilde{S}_{n, \alpha, \beta}(f)\right\|_{2} \leq C\|f\|_{2}, \quad f \in C_{\alpha, \lambda}^{2} .
\end{aligned}
$$

Proof It can be proved in a way similar to Lemma 6.

Lemma 8 For $0<t<\frac{1}{8}, \frac{t}{2} \leq x \leq 1-\frac{t}{2}, x \in[0,1], \beta<2$, we have

$$
\int_{-t / 2}^{t / 2} \delta_{n}^{-\beta}(x+u) d u \leq C(\beta) t \delta_{n}^{-\beta}(x)
$$

Lemma 9 For $0<t<\frac{1}{4}, t \leq x \leq 1-t, x \in[0,1], 0 \leq \beta \leq 2$, we have

$$
\int_{-t / 2}^{t / 2} \int_{-t / 2}^{t / 2} \delta_{n}^{-\beta}(x+u+v) d u d v \leq C t^{2} \delta_{n}^{-\beta}(x)
$$

It has been shown in [9] that Lemma 8 and Lemma 9 are valid when $\delta_{n}(t)$ is replaced by $\delta_{n}^{*}(t)$, which combining with (2.8) proves Lemma 8 and Lemma 9.

\section{Proofs of theorems}

\subsection{Proof of Theorem 1}

Define the auxiliary operators $\mathbf{S}_{n, \alpha, \beta}(f, x)$ as follows:

$$
\mathbf{S}_{n, \alpha, \beta}(f, x)=\widetilde{S}_{n, \alpha, \beta}(f, x)+L_{n, \alpha, \beta}(f, x),
$$

where

$$
L_{n, \alpha, \beta}(f, x)=f(x)-f\left(\widetilde{S}_{n, \alpha, \beta}(t, x)\right) .
$$

By (2.3) and (2.4), we have

$$
\begin{aligned}
& \left|\widetilde{S}_{n, \alpha, \beta}(t, x)-x\right| \leq \frac{C}{n}, \\
& \mathbf{S}_{n, \alpha, \beta}(1, x)=1, \quad \mathbf{S}_{n, \alpha, \beta}(t-x, x)=0,
\end{aligned}
$$

and

$$
\left\|\mathbf{S}_{n, \alpha, \beta}\right\| \leq 3 .
$$


It follows from (3.2) that

$$
\left|L_{n, \alpha, \beta}(f, x)\right| \leq \omega\left(f,\left|\widetilde{S}_{n, \alpha, \beta}(t, x)-x\right|\right) \leq C \omega\left(f, \frac{1}{n}\right) .
$$

From (1.7) and (1.8), for any fixed $x, \lambda$, and $n$, we may choose a $g_{n, x, \lambda}(t) \in \bar{D}_{\lambda}^{2}$ such that

$$
\begin{aligned}
& \|f-g\| \leq C \omega_{\varphi^{\lambda}}^{2}\left(f, n^{-1 / 2} \delta_{n}^{1-\lambda}(x)\right), \\
& \left(n^{-1 / 2} \delta_{n}^{1-\lambda}(x)\right)^{2}\left\|\varphi^{2 \lambda} g^{\prime \prime}\right\| \leq C \omega_{\varphi^{\lambda}}^{2}\left(f, n^{-1 / 2} \delta_{n}^{1-\lambda}(x)\right), \\
& \left(n^{-1 / 2} \delta_{n}^{1-\lambda}(x)\right)^{4 /(2-\lambda)}\left\|g^{\prime \prime}\right\| \leq C \omega_{\varphi^{\lambda}}^{2}\left(f, n^{-1 / 2} \delta_{n}^{1-\lambda}(x)\right) .
\end{aligned}
$$

By (3.4) and (3.6), we have

$$
\begin{aligned}
\left|\mathbf{S}_{n, \alpha, \beta}(f, x)-f(x)\right| & \leq\left|\mathbf{S}_{n, \alpha, \beta}((f-g), x)\right|+|f(x)-g(x)|+\left|\mathbf{S}_{n, \alpha, \beta}(g, x)-g(x)\right| \\
& \leq 4\|f-g\|+\left|\mathbf{S}_{n, \alpha, \beta}(g, x)-g(x)\right| \\
& \leq C \omega_{\varphi^{\lambda}}^{2}\left(f, n^{-1 / 2} \delta_{n}^{1-\lambda}(x)\right)+\left|\mathbf{S}_{n, \alpha, \beta}(g, x)-g(x)\right| .
\end{aligned}
$$

Noting that $\varphi^{2 \lambda}(x)$ and $\delta_{n}^{2 \lambda}(x)$ are concave functions on $[0,1]$, for any $t, x \in[0,1]$, and $u$ between $x$ and $t$, say $u=\theta x+(1-\theta) t, 0 \leq \theta \leq 1$, we have

$$
\begin{aligned}
& \frac{|t-u|}{\varphi^{2 \lambda}(u)}=\frac{\theta|t-x|}{\varphi^{2 \lambda}(\theta x+(1-\theta) t)} \leq \frac{\theta|t-x|}{\theta \varphi^{2 \lambda}(x)+(1-\theta) \varphi^{2 \lambda}(t)} \leq \frac{|t-x|}{\varphi^{2 \lambda}(x)}, \\
& \frac{|t-u|}{\delta_{n}^{2 \lambda}(u)} \leq \frac{|t-x|}{\delta_{n}^{2 \lambda}(x)} .
\end{aligned}
$$

By using Taylor's expansion

$$
g(t)=g(x)+g^{\prime}(x)(t-x)+\int_{x}^{t}(t-u) g^{\prime \prime}(u) d u,
$$

(3.3), and (3.11),

$$
\begin{aligned}
\left|\mathbf{S}_{n, \alpha, \beta}(g, x)-g(x)\right| & =\left|\mathbf{S}_{n, \alpha, \beta}\left(\int_{x}^{t}(t-u) g^{\prime \prime}(u) d u, x\right)\right| \\
\leq & \left|\widetilde{S}_{n, \alpha, \beta}\left(\int_{x}^{t}(t-u) g^{\prime \prime}(u) d u, x\right)\right| \\
& +\left|\int_{x}^{\widetilde{S}_{n, \alpha, \beta}(t, x)}\left(\widetilde{S}_{n, \alpha, \beta}(t, x)-u\right) g^{\prime \prime}(u) d u\right|
\end{aligned}
$$

When $x \in B_{n}$, by (2.15), (3.10), (3.2), and (2.2), we have

$$
\begin{aligned}
\left|\mathbf{S}_{n, \alpha, \beta}(g, x)-g(x)\right| & \leq C\left\|\varphi^{2 \lambda} g^{\prime \prime}\right\| \widetilde{S}_{n, \alpha, \beta}\left(\frac{(t-x)^{2}}{\varphi^{2 \lambda}(x)}, x\right)+\varphi^{-2 \lambda}(x)\left\|\varphi^{2 \lambda} g^{\prime \prime}\right\|\left(\widetilde{S}_{n, \alpha, \beta}(t, x)-x\right)^{2} \\
& \leq C n^{-1} \delta_{n}^{2-2 \lambda}(x)\left\|\varphi^{2 \lambda} g^{\prime \prime}\right\| \\
& \leq C \omega_{\varphi^{\lambda}}^{2}\left(f, n^{-1 / 2} \delta_{n}^{1-\lambda}(x)\right)
\end{aligned}
$$

where in the last inequality, (3.7) is applied. 
When $x \in B_{n}^{c}$, by (2.19), (3.10), (3.2), and (2.2), we have

$$
\begin{aligned}
\left|\mathbf{S}_{n, \alpha, \beta}(g, x)-g(x)\right| & \leq C\left\|\delta_{n}^{2 \lambda} g^{\prime \prime}\right\| \widetilde{S}_{n, \alpha, \beta}\left(\frac{(t-x)^{2}}{\delta_{n}^{2 \lambda}(x)}, x\right)+\delta_{n}^{-2 \lambda}(x)\left\|\delta_{n}^{2 \lambda} g^{\prime \prime}\right\|\left(\widetilde{S}_{n, \alpha, \beta}(t, x)-x\right)^{2} \\
& \leq C n^{-1} \delta_{n}^{2-2 \lambda}(x)\left(\left\|\varphi^{2 \lambda} g^{\prime \prime}\right\|+\frac{1}{n^{\lambda}}\left\|g^{\prime \prime}\right\|\right) \\
& \leq C n^{-1} \delta_{n}^{2-2 \lambda}(x)\left\|\varphi^{2 \lambda} g^{\prime \prime}\right\|+C\left(n^{-1 / 2} \delta_{n}^{1-\lambda}(x)\right)^{4 /(2-\lambda)}\left\|g^{\prime \prime}\right\| \\
& \leq C \omega_{\varphi^{\lambda}}^{2}\left(f, n^{-1 / 2} \delta_{n}^{1-\lambda}(x)\right)
\end{aligned}
$$

where in the last inequality, we used (3.7) and (3.8).

We complete the proof of Theorem 1 by combining (3.1), (3.5), (3.9), (3.12), and (3.13).

\subsection{Proof of Theorem 2}

With Lemma 6-Lemma 9, the proof of Theorem 2 can be found exactly in the same way as that of [9]. We omit the details here.

Competing interests

The authors declare that they have no competing interests.

\section{Authors' contributions}

The authors contributed equally to this work. All authors read and approved the final manuscript

Received: 19 August 2016 Accepted: 27 December 2016 Published online: 27 January 2017

\section{References}

1. Gadjiev, AD, Ghorbanalizaeh, AM: Approximation properties of a new type Bernstein-Stancu polynomials of one and two variables. Appl. Math. Comput. 216, 890-901 (2010)

2. Stancu, DD: Approximation of functions by a new class of linear polynomial operators. Rev. Roum. Math. Pures Appl. 13, 1173-1194 (1968)

3. Wang, ML, Yu, DS, Zhou, P: On the approximation by operators of Bernstein-Stancu types. Appl. Math. Comput. 246, 79-87 (2014)

4. İçöz, G: A Kantorovich variant of a new type Bernstein-Stancu polynomials. Appl. Math. Comput. 218, 8552-8560 (2012)

5. Taşdelen, F, Başcanbaz-Tunca, G, Erençin, A: On a new type Bernstein-Stancu operators. Fasc. Math. 48, 119-128 (2012)

6. Jung, HS, Deo, N, Dhamija, M: Pointwise approximation by Bernstein type operators in mobile interval. Appl. Math. Comput. 244, 683-694 (2014)

7. Acar, T, Aral, A, Gupta, V: On approximation properties of a new type Bernstein-Durrmeyer operators. Math. Slovaca 65, 1107-1122 (2015)

8. Ditzian, Z, Totik, V: Moduli of Smoothness. Springer, New York (1987)

9. Guo, S, Liu, L, Liu, X: The pointwise estimate for modified Bernstein operators. Studia Sci. Math. Hung. 37(1), 69-81 (2001)

\section{Submit your manuscript to a SpringerOpen ${ }^{\circ}$ journal and benefit from:}

- Convenient online submission

Rigorous peer review

- Immediate publication on acceptance

- Open access: articles freely available online

- High visibility within the field

- Retaining the copyright to your article 